Article

\title{
Surface Roughness Effects on the Broadband Reflection for Refractory Metals and Polar Dielectrics
}

\author{
Lina Cao * (iD) and Kursat Sendur *iD
}

Faculty of Engineering and Natural Science, Sabanci University, 34956 Istanbul, Turkey

* Correspondence: linairez@sabanciuniv.edu (L.C.); sendur@sabanciuniv.edu (K.S.); Tel.: +90-216-483-9527 (K.S.)

Received: 21 August 2019; Accepted: 16 September 2019; Published: 22 September 2019

\begin{abstract}
Random surface roughness and surface distortions occur inevitably because of various material processing and fabrication techniques. Tailoring and smoothing the surface roughness can be especially challenging for thermomechanically stable materials, including refractory metals, such as tungsten $(\mathrm{W})$, and polar dielectrics, such as silicon carbide ( $\mathrm{SiC})$. The spectral reflectivity and emissivity of surfaces are significantly impacted by surface roughness effects. In this paper, we numerically investigated the surface roughness effects on the spectral reflectivity and emissivity of thermomechanically stable materials. Based on our results, we determined that surface roughness effects are strongly impacted by the correlation length of the Gaussian surface. In addition, our results indicate that surface roughness effects are stronger for the materials at the epsilon-near-zero region. Surface roughness effects are stronger between the visible and infrared spectral region for $\mathrm{W}$ and around the wavelength of $12 \mu \mathrm{m}$ for $\mathrm{SiC}$, where plasma frequency and polar resonance frequency are located.
\end{abstract}

Keywords: emissivity; surface roughness effects; polar dielectric; broadband reflection; optical properties; extreme environments

\section{Introduction}

Optical structures are known for their ability to control the spectral reflectivity and emissivity of surfaces [1,2]. With the recent advances in solar thermal [3] and thermophotovoltaic [4] systems and broadband reflection/emission [5] applications, the engineering of surface structures, especially for materials that can sustain high temperature, has become essential. Deterministic surface structures, including 2D and 3D photonic crystals [6] and meta-surfaces [7], have been widely explored to tailor the spectral response of the surfaces to the incident solar spectrum [8,9], increasing the important metrics such as emission efficiency [10].

Random surface roughness and surface distortions occur inevitably as a result of material processing and fabrication techniques [11,12]. For most situations, it is hard to get a completely smooth surface. In some cases, such as the surface roughness of metals, there are reports of improvement of the solar cell energy trapping [11], and the subsequent enhancement in subwavelength imaging [13-15]. However, in many cases, the surface roughness impacts the system performance negatively. For example, it was reported that surface roughness decreases the thermal conductivity for Si nanowires [16]. As the impact of surface roughness on the system is crucial, it is important to quantify the impact of surface roughness.

Tailoring and smoothing the surface roughness can be challenging for thermomechanically stable materials, including refractory metals such as tungsten (W) and polar dielectrics, such as silicon carbide ( $\mathrm{SiC}$ ). These materials address problems like corrosion, adhesion, durability, and degraded reflectance performance due to damage, which are some issues that are encountered in various coatings [17-19]. Patterned surface structures, such as pyramidal structures [20], blazed gratings [11], 
and complex square gratings [21] of W, have been investigated by numerical approaches, such as finite difference time domain (FDTD), and experimentally demonstrated to get high absorption efficiencies. Periodic thin-film dielectric coatings deposited over refractory metals were also proposed to reduce absorption [22]. Extending such investigations of refractory metals of deterministic structures to refractory metal surfaces with random surface roughnesses is of interest. As the topology of random surface roughness is quantified by the power spectral density (PSD), the modeling and simulation of random roughness with direct numerical approaches such as FDTD or finite element method (FEM) is challenging.

In the literature, the effect of surface roughness on the interaction of electromagnetic waves with various surfaces has been investigated in detail for various other applications [23-26]. Both experimental and theoretical studies of the interaction of rough surfaces with electromagnetic waves have led to significant improvements at various spectral regions [27-34]. For example, there is a very large and established literature regarding surface roughness effects over ocean surfaces [35-38]. Complicated and sophisticated models have been developed in the context of maritime communications, and scattering of electromagnetic waves over rough ocean surfaces, originating due to environmental effects such as wind, have been investigated in detail [29,35-39]. A better understanding of surface roughness effects due to environmental factors has led to a significant improvement of wave propagation for maritime communications. Similarly, the surface roughness of soil surfaces has a profound impact on the signals and signatures of remote sensing applications involving terrestrial applications and buried objects. To understand such effects, the interaction of infrared radiation with rough soil surfaces has been studied extensively [40-43]. Surface roughness effects in solar cells have attracted significant interest, as surface texturing in solar cells has been shown to improve the efficiency of solar cells [44,45]. In addition to these studies, another area that the interaction of rough surfaces and electromagnetic waves have attracted significant interest is the study surface plasmon excitation with rough surfaces [46-52].

In terms of experimental studies and applications on rough surface scattering from thin films, there have been investigations on surface roughness effects aluminum and copper surfaces [27]. In addition, various groups have investigates surface roughness effects on plasmonic materials, such as gold and silver [46,47]. Experimental studies related to surface roughness effects in solar cells have attracted significant interest, since surface texturing in solar cells have been shown to improve the efficiency of solar cells [44,45]. In the literature, however, there is a lack of studies on the surface roughness effects of refractory metals.

Despite the established literature on rough surface scattering from surfaces, surface roughness effects on thermomechanically stable materials have been largely ignored. With the increasing interest in thermomechanically stable materials, including refractory metals and polar dielectrics, an investigation of surface roughness effect on their broadband reflection spectrum is necessary. To address these issues, in this study, we developed a model and investigated the impact of surface roughness on the spectral reflectivity under a broadband illumination. In this paper, we applied small perturbation method/small slope approximation (SPM/SSA) methods [35] to study reflectivity change in a broadband spectrum caused by Gaussian roughness for thermomechanically stable materials. The effects from the optical properties of materials and the surface roughness structures are studied and discussed. Special attention is paid to polar SiC and W.

\section{Computational Methodology}

To understand the random surface roughness effect, a rough surface configuration shown in Figure 1 is considered. Light illuminates the surface from vacuum with an incident angle, $\theta_{i}$, and azimuth angle, $\phi_{i}$. As the surface is not flat, the use of different facet angles would cause light to deflect in different directions. The directions of scattered light are thus labeled with $\theta_{s}, \phi_{s}$. To study the broadband reflectivity and emissivity, we considered normal incidence of illumination, where $\theta_{i}=0$. Surface roughness is quantified in terms of the root-mean-square (RMS) of surface height $\sigma$ and the 
transverse correlation length $l$. The power spectral density (PSD) of the surface is mathematically the Fourier transformation of the surface height self-correlation function [53]. The integration of PSD function over k-space results in the room-mean-square (RMS) of surface height, thus $\sigma$. Sinusoidal surface shapes will show as a delta function for the PSD. For a Gaussian surface, the roughness is randomly distributed with a PSD function, which follows a Gaussian distribution. In this study, we consider a Gaussian surface, the PSD of which is given as [53]

$$
W\left(k_{x}, k_{y}\right)=\frac{l^{2} \sigma^{2}}{4 \pi} e^{-\frac{\left(k_{x}^{2}+k_{y}^{2}\right) l^{2}}{4}}=\frac{l^{2} \sigma^{2}}{4 \pi} e^{-\frac{k_{\rho}^{2} l^{2}}{4}}
$$

where $l$ is transverse correlation length, $\sigma^{2}$ is the surface height variance, and $k_{\rho}^{2}=k_{x}^{2}+k_{y}^{2}$ is the wave-vector in the radial direction. In Equation (1), we assumed the same correlation length along the $\mathrm{x}$ - and $\mathrm{y}$-directions, which is $l_{x}=l_{y}$, thus the random roughness is isotropic with no azimuthal angle dependence.

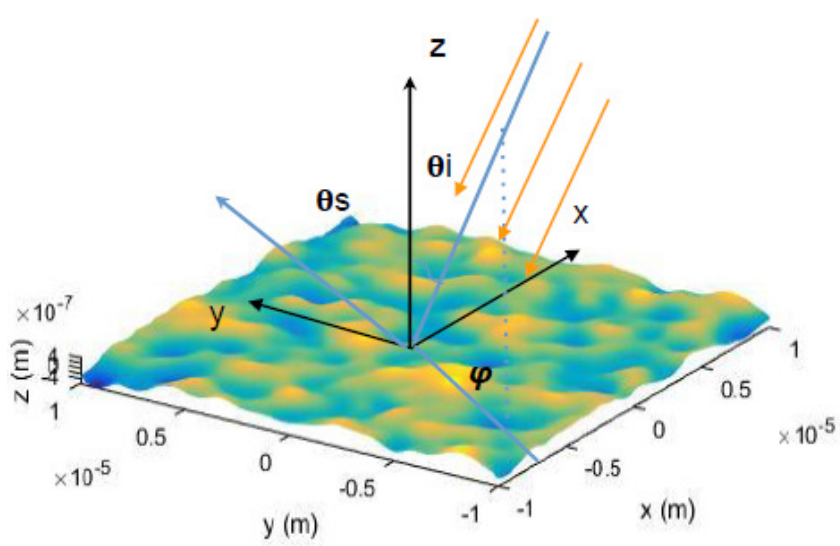

Figure 1. Schematic illustration of the surface roughness and the light scattering system.

Theoretical methods in studying the surface roughness effect includes Lippmann-Schwinger equation [54], small perturbation method [23], and small slope approximation (SSA) [35]. The numerical methods used herein include moments of momentum (MoM) and Monte Carlo [55]. Among these techniques, the SPM/SSA method, up to second order, has been proven to be valid for studying the surface roughness with small variance or small slopes. It has been widely used in the remote sensing of thermal temperature of sea surfaces [35]. In the literature, numerical techniques have been used for studying scattering from rough surfaces, including finite difference time domain (FDTD) [20], finite element method (FEM) [56], method of moments and T-Matrix methods [13-15], and Monte Carlo approaches [57]. The SPM/SSA technique used in this manuscript offers advantages for the problems that we address. Numerical approaches, such as FDTD- or FEM-based methods, provide solutions for deterministically described rough surfaces, whereas the SPM/SSA technique provides solutions for stocastically described rough surfaces. Compared to SPM/SSA, typical MoM or Monte Carlo approaches are more time-consuming. The SPM/SSA method, up to the second order, contains the scattered beam contribution from both coherent and incoherent terms, thus resumes energy conservation [58]. In this paper, we followed the formulation from the work by the authors of $[29,35]$. 
In this study, we present a theoretical investigation on rough surface scattering A simplified description of the method is discussed here. The reflection from the rough surface is given as [35]

$$
R=\left(\begin{array}{c}
\left|R_{h h}^{0}\right|^{2} \\
\left|R_{h v}^{0}\right|^{2} \\
0 \\
0
\end{array}\right)+\int_{0}^{\infty} d \beta \int_{0}^{2 \pi} d \varphi^{\prime} C\left(k_{0} \beta, \varphi^{\prime}\right)\left(\begin{array}{l}
g_{h}\left(\beta, \theta_{i}, \varphi_{i}, \epsilon, \varphi\right) \\
g_{v}\left(\beta, \theta_{i}, \varphi_{i}, \epsilon, \varphi\right) \\
g_{U}\left(\beta, \theta_{i}, \varphi_{i}, \epsilon, \varphi\right) \\
g_{V}\left(\beta, \theta_{i}, \varphi_{i}, \epsilon, \varphi\right)
\end{array}\right)
$$

where $\left|R_{h h}^{0}\right|^{2}$ and $\left|R_{h v}^{0}\right|^{2}$ corresponds to the Fresnel reflection coefficients for horizontally and vertically polarized light incidence, respectively. In Equation (2), the contributions from the $\left|R_{h h}^{0}\right|^{2}$ and $\left|R_{h v}^{0}\right|^{2}$ terms account for the reflectivity of a flat surface. Reflectivity changes due to the surface roughness are accounted in the second term in Equation (2), where $\beta=k_{\rho} / k_{0}$ and [35]

$$
C\left(k_{\rho}, \varphi\right)=k_{\rho}^{4} W\left(k_{\rho}, \varphi\right)
$$

By substituting Equation (3) into Equation (1), we obtain

$$
C\left(k_{\rho}, \varphi\right)=\frac{k_{\rho}^{4} l^{2} \sigma^{2}}{4 \pi} e^{-\frac{k_{\rho}^{2} l^{2}}{4}}
$$

As weighting functions, $g$, are functions of $\varphi_{i}-\varphi$ alone, integral over $\varphi$ makes the dependent on $\varphi_{i}$ to be vanished. In our calculations, we choose $\varphi_{i}=0$. The $g$ function in Equation (2) can be expanded into its Fourier series as

$$
g_{\gamma, n}\left(\theta_{i}, \epsilon, \beta\right)=\frac{1}{2 \pi} \int_{0}^{2 \pi} d \varphi^{\prime} e^{i n \varphi} g_{\gamma}^{\prime}\left(\theta_{i}, \epsilon, \beta, \varphi^{\prime}\right)
$$

Meanwhile, function C, given in Equation (4), can be expanded into its Fourier series as

$$
C_{n}(k \beta)=\int_{0}^{2 \pi} d \varphi^{\prime} e^{-i n \varphi^{\prime}} C\left(k \beta, \varphi^{\prime}\right)
$$

Therefore, the roughness caused reflection change can be written as

$$
\Delta R=\int_{0}^{\infty} d \beta g_{\gamma, 0}^{\prime}(\beta) C_{0}\left(k_{0} \beta\right)+2 \sum_{n=1}^{\infty} \int_{0}^{\infty} d \beta \operatorname{Re}\left\{g_{\gamma, n}^{\prime}\right\} C_{n}\left(k_{0} \beta\right)
$$

Considering the large range of wavevectors that are going to be analyzed, each azimuth harmonic term in Equation (7) is rewritten to be an linear integration over $\log _{10} \beta$, which is

$$
\int_{0}^{\infty} d \beta \operatorname{Re}\left\{g_{\gamma, n}^{\prime}(\beta)\right\} C_{n}\left(k_{0} \beta\right)=(\ln 10) \int_{-\infty}^{\infty} d\left(\log _{10} \beta\right) \beta \operatorname{Re}\left\{g_{\gamma, n}^{\prime}(\beta)\right\} C_{n}\left(k_{0} \beta\right)
$$

In the following sections, the surface roughness-caused reflection change $\Delta R$ is calculated using Equation (7) and Equation (8). In Equation (7), $\Delta R$ is decomposed into two terms, $g_{\gamma, n}^{\prime}(\beta)$ and $C_{n}\left(k_{0} \beta\right)$, given by Equation (5) and Equation (6), respectively. Here, the weighing function, $g$, is determined by the material optical properties, and $C$ depends only on the surface profiles. The magnitude of $g$ values are derived from scattering coefficients; the full description is illustrated in the work by the authors of [29].

\section{Results and Discussions}

As the PSD of sinusoidal surfaces are delta functions at the corresponding wave-vector $k$ [53], each value of the weighting function $g$ at a given $k$ determines the reflection change caused by the corresponding sinusoidal surface. For the Gaussian surface in this paper, we take the 0 th harmonic 
of the weighting function into account. As a result, weighting functions depend only on the optical permittivity of materials for a chosen beam incident angle. To illustrate the different scattering properties of different materials in this study, the weighting functions for materials with artificial dielectric constants are presented. Refractive index of material is represented as $n+i k$, where $n$ and $k$ correspond to the real and imaginary parts of the dielectric constant, respectively.

Figure 2 shows the normal incidence weighting functions for dielectrics with $k=0$, where the materials are transparent with no absorption. As can be seen, there is a strong resonance peak at $\beta=1$; a secondary resonance peak happens around the $\beta=n$. At $\beta=1$, the corresponding space periodicity of the surface has the same periodicity of incident wavelength $\lambda$, where perfect phasing matching is provided for the reflected waved, therefore, causing less reflection. For the secondary resonance peak, the corresponding periodicity is $\lambda / n$, which is the wavelength of beam inside the material. As there is no absorption from the material, reflection changes are purely brought by the roughness periodicity-caused momentum change. When the surface roughness gets larger than the incident wavelength, it behaves like smooth surface and weighting function magnitude gets close to 0 . Similarly, when the surface roughness gets very small, the weighting function also decreases. Surface roughness with the periodicity between incident wavelength and the wavelength in the material have strong effect of the surface reflection and the effect slowly decreases as roughness gets smaller.

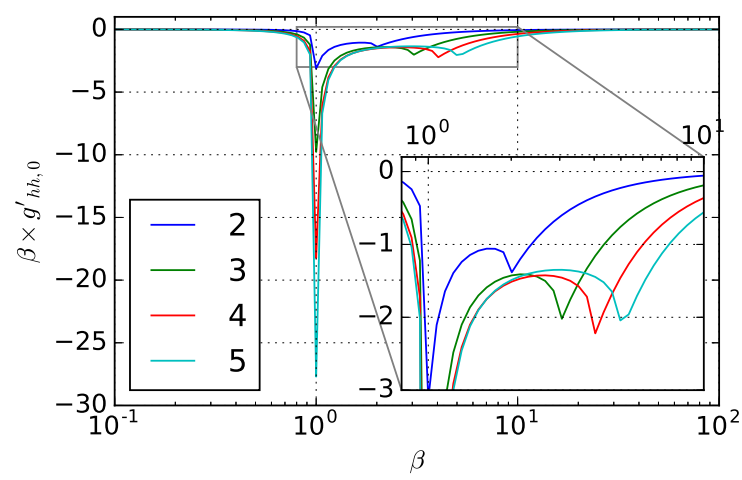

Figure 2. 0th-order weighting function magnitude with respect to optical constant for materials with $k=0$. The legend in the figure shows the corresponding $n$ value for each curve. Inset plot corresponds to the region in the gray box.

The materials experience absorption of the penetrated beam, which can be quantified by the imaginary part of permittivity, $\epsilon=n^{2}=n^{2}-k^{2}+2 n k i$, at $n=k, \epsilon=0$. For metals, this is caused by the electron oscillations and the corresponding frequency is called plasma frequency $\omega_{p}$. For polar dielectrics, phonon resonances can also cause an epsilon-near-zero region, which corresponds to the so-called Reststrahlen band [59]. Here, artificial material with fixed $n_{r}=3$ is chosen and the corresponding weighting functions for varying $k$ values are calculated and plotted in Figure 3 . The blue curve corresponds to $k=0$, where two resonance peaks at the locations of $\lambda$ and $\lambda / n$ exist in the curve as discussed above. As $k$ increases, beams that have penetrated the material experience energy absorption, providing extra phase and momentum change of optical beams; therefore, the second resonance peak which corresponds to the light wavelength inside the material decreases and disappears. Due to the absorption and reemission, the reflected beams experience more complicated phase change compared to pure dielectrics with $k=0$. As $k$ increases and becomes larger than $n_{r}, \epsilon$ becomes negative. Surface behaves like perfect electrical conductor with high reflectivity, the influence from surface roughness under this condition is also relatively small. Weighting function shows as one sharp peak at the incident $\lambda$. 


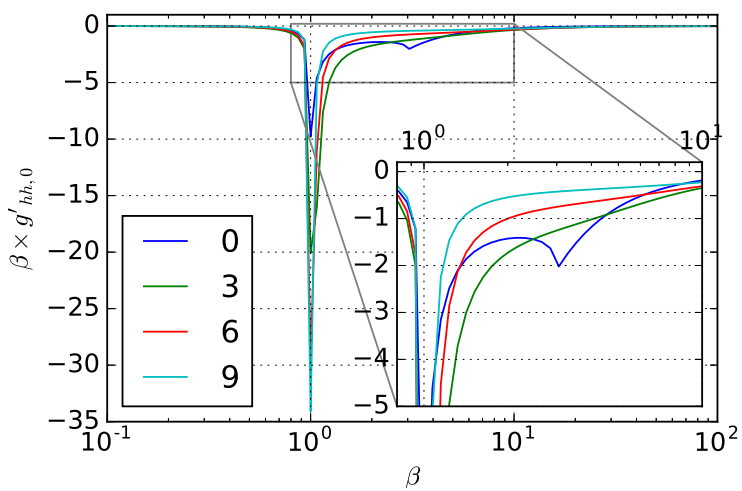

Figure 3. 0th-order weighting function magnitude with respect to dielectric constant for materials with $n_{r}=3$. The legend in the figure shows the corresponding $k$ value for each curve. Inset plot corresponds to the boxed region.

Considering function $C(\beta)$, the maximum magnitude happens at $\frac{\partial C}{\partial \beta}=0$, where $\frac{2 \sqrt{2}}{k_{0} l}=\frac{\sqrt{2}}{\pi} \frac{\lambda_{0}}{l}$ and the maximum value is defined as

$$
C_{\max }=\frac{16}{\pi e^{2}}\left(\frac{\sigma}{l}\right)^{2}
$$

Thus, the maximum value of $C$ for Gaussian surfaces is proportional to $\left(\frac{\sigma}{l}\right)^{2}$. In Figure 4 , we plotted four $C$ functions with the transverse correlation lengths chosen to be $\lambda / 10, \lambda / 5, \lambda / 2.5, \lambda$, respectively. For all these curves, $\sigma=l / 5 . \sigma / l$ are kept to be the same thus the $C$ curves have the same maximum values according to Equation (9). $\sigma / l$ shows the general slope of the surface roughness, for SPM/SSA method to be valid, $\sigma / l$ of smaller than 4 is necessary [58]. As illustrated in Figure 4, the peak positions of function $C$ moves to the smaller length scales section as $l$ gets smaller. At the location $\beta=1$, which is the resonance position for weighting functions, the maximum $C$ happens at $l / \lambda_{0}=\pi / \sqrt{2} \approx 2.22$. Due to the fast fall off of the weighting functions, when $l=\lambda_{0}$ (cyan curve in Figure 4), panel $(b)$ overlaps between the function $C$, and the weighing function becomes too small, thus the reflection change caused by the roughness can be neglected. In other words, the roughness length scale is too big for the incident light to detect and the reflection value is close to smooth flat surface. When $l$ is less than $\lambda_{0}$, the weighting functions do not fall as fast, and the maximum overlap between these two function happens when 1 is between $\lambda / n$ and $\lambda / 2.22$. Panel (c) in Figure 4 plotted the reflection change for Gaussian surfaces with different correlation lengths. The $x$-axis is the correlation length $l$ in the unit of $\lambda / 2.22$, in other words, the location of $C_{\max }$ for surface curves. As illustrated, the maximum $\Delta R$ happens with the maximum overlap between the surface $C$ function and the weighting function, which is in between $\lambda / n$ and $\lambda / 2.22$. Two example surface height profiles are shown in Figure 4, panel (a). At the left profile, panel $a(1), l=\lambda / 2.5=600 \mathrm{~nm}$, and for the right profile $l=\lambda / 10=150 \mathrm{~nm}$. The $C$ function for these two surface profiles are the dotted green and dotted red curve respectively. As stated previously, $\sigma / l$ are kept the same for these illustrations, therefore, smaller $l$ corresponds to smaller defects.

After a brief discussion of the weighting function and the correlation functions, we discuss the surface roughness effects for refractory metals and polar dielectrics, which are popular materials for high temperature applications. Here, we chose tungsten and $\mathrm{SiC}$ as two examples, and analyzed the surface reflection change caused by random surface roughness.

Similar to other refractory metals, $\mathrm{W}$ shows a high reflection at far-infrared range, whereas it experiences a considerable absorption in the visible and near-infrared [22]. We pick four wavelengths: $500 \mathrm{~nm}, 1000 \mathrm{~nm}, 1500 \mathrm{~nm}$, and $2000 \mathrm{~nm}$. We calculated the corresponding weighting functions, which are plotted in the top panel of Figure 5. With reference to the middle panel of Figure 5, at $\lambda=500 \mathrm{~nm}, n>k, \epsilon>0$; at $\lambda=1000 \mathrm{~nm}, n$ gets smaller compared to $500 \mathrm{~nm}, k$ is bigger, and $\epsilon$ gets close to 0 . As can be seen from the second panel, the weighting function for $\lambda=500 \mathrm{~nm}, 1000 \mathrm{~nm}$ is 
very close in magnitude. At $\lambda=1500 \mathrm{~nm}$, the magnitude of the weighting function starts to get smaller especially when $\beta$ gets away from the incident wavelength resonance location. At $\lambda=2000 \mathrm{~nm}, \epsilon$ gets far negative, where weighting function gets a lot smaller in magnitude compared to the previous three wavelengths. As the maximum reflection change point is closely correlated with the incident $\lambda$, if we look at the problem from the broadband spectrum point, for a fixed topology of surface roughness, its influence on the spectrum will mainly be around the $l / 2.22$ location. Keeping this idea in mind, the broadband spectrum for W Gaussian surfaces are calculated and plotted in the bottom panel. The legend in the panel shows the maximum location of the chosen $C$ function, in other words, $l / 2.22$. For the first three curves- $l / 2.22=500 \mathrm{~nm}, 1000 \mathrm{~nm}$, and $1500 \mathrm{~nm}$-the maximum $\Delta R$ value location is around the corresponding wavelengths. For all of these curves, $\sigma / l$ are kept the same for each surface profile, therefore $\sigma$ will be larger for bigger $l$ curves. It shows in the curve that the $\Delta R$ values smooth out at longer wavelengths. This confirms that when the surface roughness size is very small compared to $\lambda, \Delta R$ depends only on the value of $\sigma$ [60]. For the red curve, where $C_{\max }$ location at $2000 \mathrm{~nm}$, it is expected that one resonance peak would show around $\lambda=2000 \mathrm{~nm}$, however, the resonance peak actually appears at around $\lambda=1500 \mathrm{~nm}$, which is a big resonance shift. Referring to the first three curves, we also see shoulder peaks at around $\lambda=1500 \mathrm{~nm}$. Refer to the top panel in this figure, for Tungsten, epsilon becomes negative at around $\lambda>1000 \mathrm{~nm}$, where surface plasmon resonances (SPR) can be excited. The localized surface plasmon resonance will increase the absorption of the material thus shows as a higher reflection reduction. Gaussian surfaces with one certain transverse correlation length can be decomposed into sinusoidal surfaces with the corresponding periodicity. As the corresponded periodicities for Gaussian surface covers one certain range centered around $l$, shown as the $C$ function in Figure 4 , if $l$ is around $\omega_{p}$, LSPR gets excited and increases absorption. At $C_{\max }$ location at $2000 \mathrm{~nm}$, the weighting function for $\lambda=2000 \mathrm{~nm}$ is relatively small due to the high negative number of $\epsilon$, the resonance peak matching the periodicity does not get as high as the LSPR peak, thus showing as a resonance shift on the reflection spectrum. As $l$ gets larger and further away from the $\omega_{p}$ frequency, corresponded LSPR resonance also gets lower due to the magnitude of $C$ function at this resonance frequency gets lower. The LSPR resonance peak can still be seen at $C_{\max }=2500 \mathrm{~nm}$, but disappears at $C_{\max }=3000 \mathrm{~nm}$. At these larger $l \mathrm{~s}$, the primary resonance peak is also not as sharp and it falls very fast to one smooth value.

A similar analysis is performed for $\mathrm{SiC}$. Figure 6 illustrates the optical constant, the weighting functions of the selected wavelengths, as well as the broadband reflection change for fixed Gaussian surface topologies. $\mathrm{SiC}$ is a lossy dielectric in infrared range. Incident beams penetrates dielectrics thus resulting low reflection. Four wavelengths $-\lambda=410 \mathrm{~nm}, 1500 \mathrm{~nm}, 11,700 \mathrm{~nm}$, and 24,900 nm-were chosen to illustrate the weighting functions, as shown in the top panel of Figure 6. Compared to $\mathrm{W}$, the magnitude of weighing functions are smaller. As the magnitude of weighting function of $\mathrm{W}$ approaches -20 , the magnitude for $\mathrm{SiC}$ is -1 . Even the $n$ values for $\mathrm{W}$ and $\mathrm{SiC}$ are comparable, as there is significant difference in $k$ values. Lower $k$ values for $\mathrm{SiC}$ means lower absorption for penetrated beams propagating inside the material. As seen in the top panel, the secondary resonance peak shows for the chosen wavelengths. As the differences among the weighting functions are relatively small, roughness caused reflection change are also highly depended on the ration of $l / \lambda$, with $\sigma / l$ fixed. The results are shown in panel 3. As expected, at the long wavelength range, the surface reflection change increases as $\sigma$ increases. The maximum reflection change occurs when the maximum overlap of the Gaussian surface $C$ function and weighting function, which is when the $C_{\max }$ locates at $\lambda / 2.22$. Further, a secondary resonance peak is located around the location of $\lambda$ between 10,000 nm and $15000 \mathrm{~nm}$, where the lattice oscillations cause extra absorption. These lattice polar resonances behave like Lorentz oscillators for polar dielectric crystals, and the dispersion relation are similar to those of metals [59]. Referring to middle panel, which illustrates the optical constant of SiC, we can clearly see one resonance peak at $\lambda \approx 10 \mu \mathrm{m}-15 \mu \mathrm{m}$. For some polar dielectrics, such as $4 \mathrm{H}-\mathrm{SiC}$, surface phonon resonance $(\mathrm{SPhR})$ modes can be excited during the Reststrahlen band, where results epsilon near to zero 
and negative $\operatorname{Re}(\epsilon)$. According to our previous discussion, the surface roughness for these materials will be expected to experience some higher reflection change at the resonant frequency location.

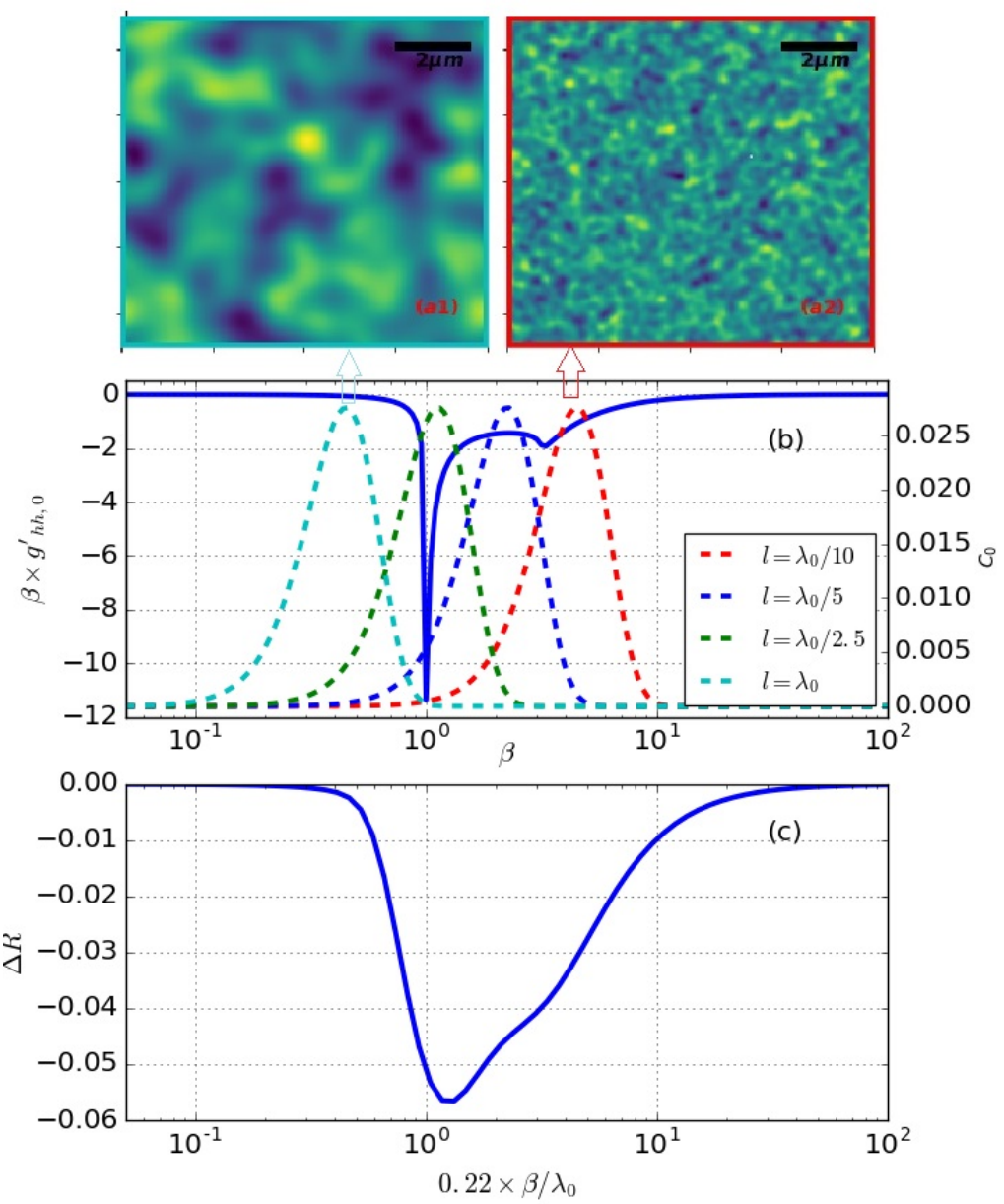

Figure 4. Illustration of $C_{0}$ functions for Gaussian surfaces overlapped with the weighting function and corresponding surface profile. The top panels $(\mathbf{a 1}, \mathbf{a} 2)$ show two example surface height profiles. Panel (b) shows weighting function with four $C_{0}$ functions. Panel (c) shows roughness caused surface reflection change $\Delta R$ versus the $0.22 \beta / l$, which is the location of $C_{0}$ function maximum.

After this detailed discussion on various materials, the main findings and differences of each case can be summarized as follows. For the lossless case, the weighting function has two resonance peaks, corresponding to the incident wavelength and wavelength of beam inside the material, however the magnitude of weighting functions are limited compared to lossy materials. For the lossy materials that we studied, a comparison of $\mathrm{W}$ and $\mathrm{SiC}$ suggests that $\mathrm{SiC}$ is more dielectric as $n>k$ for interested spectrum range for $\mathrm{SiC}$ ( $400 \mathrm{~nm}$ to $2 \mathrm{um}$ ); thus, for the same $\sigma$, which is height variance, roughness causes bigger reflection change for $W$, especially at the near-infrared range, where epsilon of $W$ is close to 0 . For $\mathrm{SiC}$, the roughness caused by the spectrum change can show the magnification in the $10 \mathrm{um}$ to $15 \mathrm{um}$ range, which corresponds to the phonon resonance in $\mathrm{SiC}$; however, as $\mathrm{SiC}$ is still dielectric at this range, the magnification is limited. 

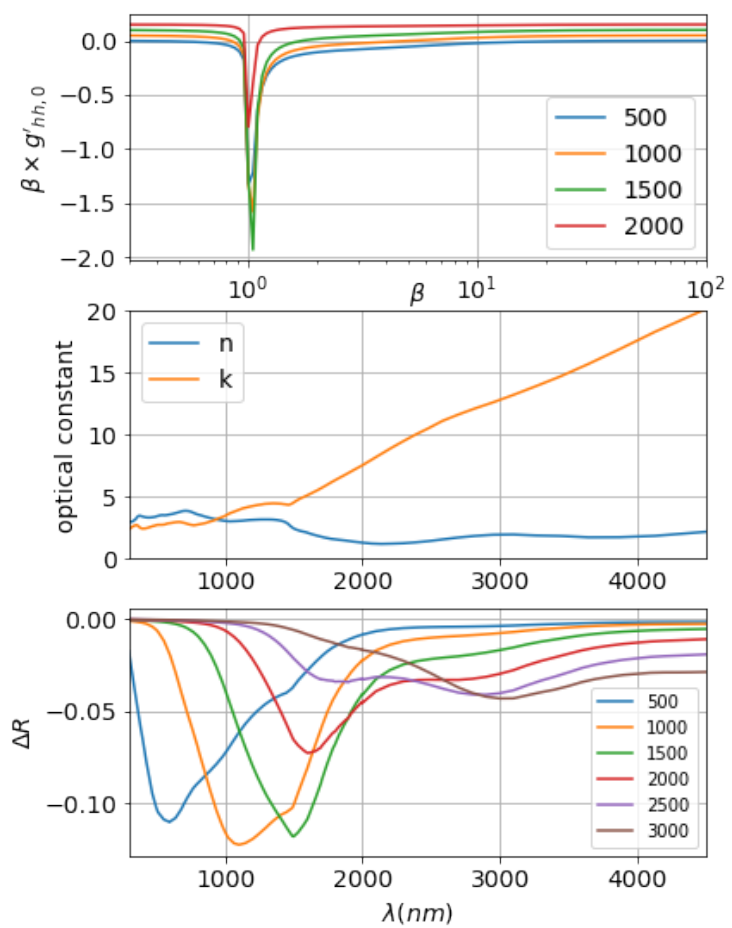

Figure 5. Random roughness effects on tungsten surface. Top panel: weighting function for wavelengths $\lambda=500 \mathrm{~nm}, 1000 \mathrm{~nm}, 1500 \mathrm{~nm}$, and $2000 \mathrm{~nm}$. Middle panel: optical dielectric constants for $\mathrm{W}$ at the visible and near-infrared range. Bottom panel: Reflection spectrum change of W surface for Gaussian surface with different correlation lengths. Legend: $2.22 \mathrm{in} \mathrm{nm}$.
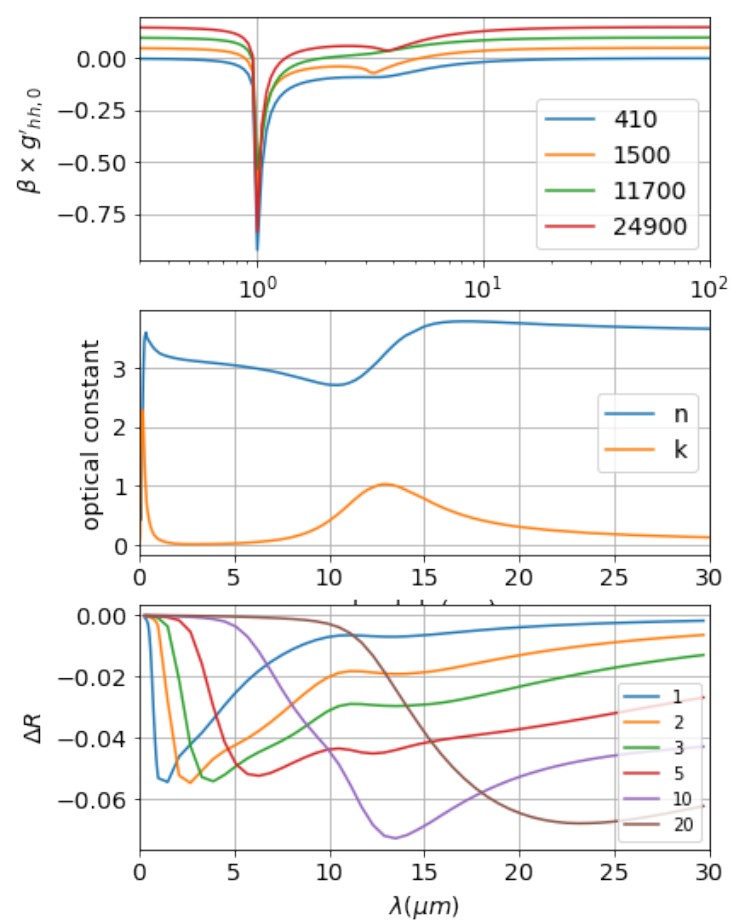

Figure 6. Random roughness effects on $\mathrm{SiC}$ surface. Top panel: weighting function for wavelengths $\lambda=410 \mathrm{~nm}, 1500 \mathrm{~nm}, 11,700 \mathrm{~nm}, 24,900 \mathrm{~nm}$. Middle panel: optical dielectric constants for SiC at the visible and near-infrared range. Bottom panel: Reflection spectrum change of $\mathrm{SiC}$ surface for Gaussian surface with different correlation lengths. Legend: $2.22 l$ in $\mu \mathrm{m}$. 


\section{Conclusions}

In this paper, we applied the SPM/SSA method to study the random surface roughness effects on the reflectivity of refractory metals and polar dielectrics. This method enables the decomposition of surface roughness topography and material properties. Larger values of $n$ show a higher roughness effect as well as a broader length scale range, which are illustrated as weighting functions. Transparent dielectrics with low $k$ values experience two resonance locations on the weighting function, corresponding to the periodicity of roughness same as incident wavelength and the wavelength in material. The absorption increase ( $k$ increase) for materials will increase the weighting function in magnitude, but the secondary resonance peak weakens and disappears. The maximum roughness effect happens when $k$ is similar, in terms of magnitude, to $n$, where epsilon is close to zero.

For refractory metal $\mathrm{W}$, as $n$ and $k$ are relatively close in terms of magnitude at the visible to infrared range, surface roughnesses with the corresponding correlation lengths cause higher absorption change with same roughness height variance. At the far-infrared region, where flat W surface experience high reflectivity, the reflection change due to surface roughness is also limited. For $\mathrm{SiC}$, as the magnitude $k$ is smaller than $n$ at infrared, surface roughness caused reflection change is limited; however, polar resonance at $\lambda$ around $12 \mu \mathrm{m}$ causes one resonance peak at this location for the surface roughness effect. Our results indicate that maximum impact on the reflection from a rough material surface is observed when the correlation length of the random surface roughness satisfies $l=2.22 \lambda_{0}$ condition and the operation frequency matches the internal absorption frequencies (plasma frequency and phonon frequency).

Author Contributions: Both authors contributed equally to the methodology, writing, and revising of the article. Coding the theoretical formulas and performing the numerical simulations, L.C. Funding acquisition and project administration, K.S.

Funding: This material is based on work supported by the Air Force Office of Scientific Research (Aerospace Materials for Extreme Environments Program, PM: Ali Sayir) under award number FA9550-18-1-9240.

Conflicts of Interest: The authors declare no conflicts of interest.

\section{References}

1. Gupta, M.C.; Ungaro, C.; Foley, J.J.; Gray, S.K. Optical nanostructures design, fabrication, and applications for solar/thermal energy conversion. Sol. Energy 2018, 165, 100-114. [CrossRef]

2. Inoue, T.; De Zoysa, M.; Asano, T.; Noda, S. Realization of narrowband thermal emission with optical nanostructures. Optica 2015, 2, 27-35. [CrossRef]

3. Tian, Y.; Zhao, C.Y. A review of solar collectors and thermal energy storage in solar thermal applications. Appl. Energy 2013, 104, 538-553. [CrossRef]

4. Ferrari, C.; Melino, F.; Pinelli, M.; Spina, P.; Venturini, M. Overview and status of thermophotovoltaic systems. Energy Procedia 2014, 45, 160-169. [CrossRef]

5. Huang, Y.F.; Chattopadhyay, S.; Jen, Y.J.; Peng, C.Y.; Liu, T.A.; Hsu, Y.K.; Pan, C.L.; Lo, H.C.; Hsu, C.H.; Chang, Y.H.; et al. Improved broadband and quasi-omnidirectional anti-reflection properties with biomimetic silicon nanostructures. Nat. Nanotechnol. 2007, 2, 770. [CrossRef]

6. Fleming, J.; Lin, S.; El-Kady, I.; Biswas, R.; Ho, K. All-metallic three-dimensional photonic crystals with a large infrared bandgap. Nature 2002, 417, 52. [CrossRef] [PubMed]

7. Liu, Y.; Zhang, X. Metamaterials: A new frontier of science and technology. Chem. Soc. Rev. 2011, 40, 2494-2507. [CrossRef]

8. Zhou, Z.; Sakr, E.; Sun, Y.; Bermel, P. Solar thermophotovoltaics: Reshaping the solar spectrum. Nanophotonics 2016, 5, 1-21. [CrossRef]

9. Khodasevych, I.E.; Wang, L.; Mitchell, A.; Rosengarten, G. Micro-and nanostructured surfaces for selective solar absorption. Adv. Opt. Mater. 2015, 3, 852-881. [CrossRef]

10. Oh, J.; Yuan, H.C.; Branz, H.M. An 18.2\%-efficient black-silicon solar cell achieved through control of carrier recombination in nanostructures. Nat. Nanotechnol. 2012, 7, 743. [CrossRef] 
11. Ungaro, C.; Gray, S.K.; Gupta, M.C. Black tungsten for solar power generation. Appl. Phys. Lett. 2013, 103, 071105. [CrossRef]

12. Zhang, S.; To, S.; Wang, S.; Zhu, Z. A review of surface roughness generation in ultra-precision machining. Int. J. Mach. Tools Manuf. 2015, 91, 76-95. [CrossRef]

13. Bagley, J.Q.; Tsang, L.; Ding, K.H.; Ishimaru, A. Optical transmission through a plasmon film lens with small roughness: Enhanced spatial resolution of images of single source and multiple sources. JOSA B 2011, 28, 1766-1777. [CrossRef]

14. Wang, H.; Bagley, J.Q.; Tsang, L.; Huang, S.; Ding, K.H.; Ishimaru, A. Image enhancement for flat and rough film plasmon superlenses by adding loss. JOSA B 2011, 28, 2499-2509. [CrossRef]

15. Huang, S.; Wang, H.; Ding, K.H.; Tsang, L. Subwavelength imaging enhancement through a three-dimensional plasmon superlens with rough surface. Opt. Lett. 2012, 37, 1295-1297. [CrossRef]

16. Lim, J.; Hippalgaonkar, K.; Andrews, S.C.; Majumdar, A.; Yang, P. Quantifying surface roughness effects on phonon transport in silicon nanowires. Nano Lett. 2012, 12, 2475-2482. [CrossRef] [PubMed]

17. Mishra, S.K.; Kumar, V.; Tiwari, S.; Mishra, T.; Angula, G.; Adhikari, S. Development and degradation behavior of protective multilayer coatings for aluminum reflectors for solar thermal applications. Thin Solid Films 2016, 619, 202-207. [CrossRef]

18. Fryauf, D.M.; Phillips, A.C.; Kobayashi, N.P. Corrosion protection of silver-based telescope mirrors using evaporated anti-oxidation overlayers and aluminum oxide films by atomic layer deposition. Proc. SPIE 2016, 9924, 99240S.

19. Fryauf, D.M.; Phillips, A.C.; Kobayashi, N.P. Corrosion barriers for silver-based telescope mirrors: Comparative study of plasma-enhanced atomic layer deposition and reactive evaporation of aluminum oxide. J. Astron. Telescopes Instrum. Syst. 2015, 1, 044002. [CrossRef]

20. Rephaeli, E.; Fan, S. Tungsten black absorber for solar light with wide angular operation range. Appl. Phys. Lett. 2008, 92, 211107. [CrossRef]

21. Chen, Y.B.; Zhang, Z. Design of tungsten complex gratings for thermophotovoltaic radiators. Opt. Commun. 2007, 269, 411-417. [CrossRef]

22. Keçebaş, M.A.; Şendur, K. Enhancing the spectral reflectance of refractory metals by multilayer optical thin-film coatings. JOSA B 2018, 35, 1845-1853. [CrossRef]

23. Voronovich, A.G. Wave Scattering from Rough Surfaces; Springer: Berlin/Heidelberg, Germany, 1994.

24. Beckmann, P.; Spizzichino, A. The Scattering Of Electromagnetic Waves From Rough Surfaces; Artech House: Norwood, MA, USA, 1987.

25. Ogilvy, J.A. Theory of Wave Scattering From Random Rough Surfaces; CRC Press: Boca Raton, FL, USA, 1991.

26. Torrance, K.E.; Sparrow, E.M. Theory for Off-Specular Reflection From Roughened Surfaces. J. Opt. Soc. Am. 1967, 57, 1105-1114. [CrossRef]

27. Videen, G.; Hsu, J.; Bickel, W.; Wolfe, W. Polarized light scattering from rough surfaces. J. Opt. Soc. Am. 1992, 9, 1111-1118. [CrossRef]

28. Sinha, S.K.; Sirota, E.B.; Garoff, S.; Stanley, H.B. X-ray and neutron scattering from rough surfaces. Phys. Rev. B 1988, 38, 2297-2311. [CrossRef]

29. Yueh, S.H.; Kwok, R.; Li, F.; Nghiem, S.; Wilson, W.; Kong, J.A. Polarimetric passive remote sensing of ocean wind vectors. Radio Sci. 1994, 29, 799-814. [CrossRef]

30. Yueh, H.A.; Shin, R.T.; Kong, J.A. Scattering of electromagnetic waves from a periodic surface with random roughness. J. Appl. Phys. 1988, 64, 1657-1670. [CrossRef]

31. Yueh, S.H.; Kwok, R. Electromagnetic fluctuations for anisotropic media and the generalized Kirchhoff's law. Radio Sci. 1993, 28, 471-480. [CrossRef]

32. Church, E.; Jenkinson, H.; Zavada, J. Relationship between surface scattering and microtopographic features. Opt. Eng. 1979, 18, 182125. [CrossRef]

33. Hottel, H.C.; Sarofim, A.F. Radiative Transfer; McGraw-Hill, New York, NY, USA, 1967.

34. Tsang, L.; Kong, J.A.; Shin, R.T. Theory of Microwave Remote Sensing; Wiley-Interscience: New York, NY, USA, 1985.

35. Johnson, J.T.; Zhang, M. Theoretical study of the small slope approximation for ocean polarimetric thermal emission. IEEE Trans. Geosci. Remote Sens. 1999, 37, 2305-2316. [CrossRef]

36. Chou, H.T.; Johnson, J.T. A novel acceleration algorithm for the computation of scattering from rough surfaces with the forward-backward method. Radio Sci. 1998, 33, 1277-1287. [CrossRef] 
37. Johnson, J.T. A numerical study of scattering from an object above a rough surface. IEEE Trans. Antennas Propagation 2002, 50, 1361-1367. [CrossRef]

38. Pino, M.R.; Landesa, L.; Rodriguez, J.L.; Obelleiro, F.; Burkholder, R.J. The generalized forward-backward method for analyzing the scattering from targets on ocean-like rough surfaces. IEEE Trans. Antennas Propagation 1999, 47, 961-969. [CrossRef]

39. Zavorotny, V.U.; Voronovich, A.G. Scattering of GPS signals from the ocean with wind remote sensing application. IEEE Trans. Geosci. Remote Sens. 2000, 38, 951-964. [CrossRef]

40. Sendur, I.K.; Baertlein, B.A. Numerical simulation of thermal signatures of buried mines over a diurnal cycle. Proc. SPIE 2000, 4038, 56-167.

41. Sendur, I.K.; Johnson, J.T.; Baertlein, B.A. Analysis of polarimetric IR phenomena for detection of surface mines. Proc. SPIE 2001, 4394, 53-163.

42. Oh, Y.; Sarabandi, K.; Ulaby, F.T. An empirical model and an inversion technique for radar scattering from bare soil surfaces. IEEE Trans. Geosci. Remote Sens. 1992, 30, 370-381. [CrossRef]

43. Choudhury, B.J.; Schmugge, T.J.; Chang, A.; Newton, R.W. Effect of surface roughness on the microwave emission from soils. J. Geophys. Res.: Oceans 1979, 84, 5699-5706. [CrossRef]

44. Zheng, L.; Ma, Y.; Chu, S.; Wang, S.; Qu, B.; Xiao, L.; Chen, Z.; Gong, Q.; Wu, Z.; Hou, X. Improved light absorption and charge transport for perovskite solar cells with rough interfaces by sequential deposition. Nanoscale 2014, 6, 8171-8176. [CrossRef]

45. Fä̈, S.; Feitknecht, L.; Schlüchter, R.; Kroll, U.; Vallat-Sauvain, E.; Shah, A. Rough ZnO layers by LP-CVD process and their effect in improving performances of amorphous and microcrystalline silicon solar cells. Sol. Energy Mater. Solar Cells 2006, 90, 2960-2967. [CrossRef]

46. Raether, H. Surface Plasmons on Smooth and Rough Surfaces and on Gratings; Springer: Berlin/Heidelberg, Germany, 1988.

47. Hoffmann, A.; Lenkefi, Z.; Szentirmay, Z. Effect of roughness on surface plasmon scattering in gold films. J. Phys.: Condensed Matter 1998, 10, 5503-5513. [CrossRef]

48. Kanso, M.; Cuenot, S.; Louarn, G. Roughness effect on the SPR measurements for an optical fibre configuration: Experimental and numerical approaches. J. Opt. A: Pure Appl. Opt. 2007, 9, 586-592. [CrossRef]

49. Farias, G.A.; Maradudin, A.A. Surface plasmons on a randomly rough surface. Phys. Rev. B 1983, 28, 5675-5687. [CrossRef]

50. Maradudin, A.A.; Mills, D.L. Scattering and absorption of electromagnetic radiation by a semi-infinite medium in the presence of surface roughness. Phys. Rev. B 1975, 11, 1392-1415. [CrossRef]

51. Celli, V.; Maradudin, A.A.; Marvin, A.M.; McGurn, A.R. Some aspects of light scattering from a randomly rough metal surface. J. Opt. Soc. Am. A 1985, 2, 2225-2239. [CrossRef]

52. Simonsen, I.; Maradudin, A.A. Numerical simulation of electromagnetic wave scattering from planar dielectric films deposited on rough perfectly conducting substrates. Opt. Commun. 1999, 162, 99-111. [CrossRef]

53. Jacobs, T.D.; Junge, T.; Pastewka, L. Quantitative characterization of surface topography using spectral analysis. Surface Topography: Metrol. Proper. 2017, 5, 013001. [CrossRef]

54. Tsang, L.; Ding, K.H.; Li, X.; Duvelle, P.N.; Vella, J.H.; Goldsmith, J.; Devlin, C.L.; Limberopoulos, N.I. Studies of the influence of deep subwavelength surface roughness on fields of plasmonic thin film based on Lippmann-Schwinger equation in the spectral domain. JOSA B 2015, 32, 878-891. [CrossRef]

55. Sadiku, M.N. Numerical Techniques in Electromagnetics; CRC Press: Boca Raton, FL, USA, 2000.

56. Canteli, D.; López, J.; Lauzurica, S.; Lluscà, M.; Sánchez-Aniorte, M.; Bertomeu, J.; Morales, M.; Molpeceres, C. Analysis by Finite Element Calculations of Light Scattering in Laser-textured AZO Films for PV thin-film Solar Cells. Energy Procedia 2015, 84, 78-85. [CrossRef]

57. Delacrétaz, Y.; Seydoux, O.; Chamot, S.; Ettemeyer, A.; Depeursinge, C. Monte Carlo simulation of the field back-scattered from rough surfaces. J. Opt. Soc. Am. A 2012, 29, 270-277. [CrossRef]

58. Wang, T.; Tsang, L.; Johnson, J.T.; Tan, S. Scattering and transmission of waves in multiple random rough surfaces: Energy conservation studies with the second order small perturbation method. Progress Electromagn. Res. 2016, 157, 1-20. [CrossRef] 
59. Caldwell, J.D.; Lindsay, L.; Giannini, V.; Vurgaftman, I.; Reinecke, T.L.; Maier, S.A.; Glembocki, O.J. Low-loss, infrared and terahertz nanophotonics using surface phonon polaritons. Nanophotonics 2015, 4, 44-68. [CrossRef]

60. Bennett, H.; Porteus, J. Relation between surface roughness and specular reflectance at normal incidence. JOSA 1961, 51, 123-129. [CrossRef]

(C) 2018 by the authors. Licensee MDPI, Basel, Switzerland. This article is an open access article distributed under the terms and conditions of the Creative Commons Attribution (CC BY) license (http:/ / creativecommons.org/licenses/by/4.0/). 